\title{
Mejora de la ubicación del centro articular de un sistema de captura de movimiento sin marcadores a través de un método de calibración funcional: estudio piloto
}

\section{Improving the joint centre location of a markerless motion analysis system through a functional calibration method: a pilot study}

\section{Doctorando:}

\section{Magalí Sganga}

Universidad Maimónides - Argentina

sgangamagali@gmail.com

\section{Director/es:}

\section{Lucas Eduardo Ritacco}

\section{Co-director/es:}

\section{Emiliano Pablo Ravera}

\section{Resumen}

El análisis biomecánico es una herramienta para evaluar movimientos patológicos y su rehabilitación. Esta investigación estudia la factibilidad de mejoras en la ubicación del centro articular de cadera en un Sistema de Captura de Movimiento sin Marcadores a través de la aplicación de un método de calibración funcional. Se analizó el movimiento de una joven mujer sin impedimentos físicos. Se adquirió simultáneamente información del sistema gold standard (Motion Capture System, MOCAP) y una alternativa de bajo costo (Microsoft Kinect). Se grabaron adquisiciones estáticas y dinámicas. A partir de la información obtenida con Kinect, se crearon cinco marcadores virtuales para cada pierna y se introdujeron en optimal common shape technique y en symmetrical centre of rotation estimation method para determinar los centros articulares de ambas caderas. Los resultados mostraron mejora en la ubicación del centro de rotación cuando fueron comparados con la información de MOCAP. En conclusión, este enfoque podría mejorar la información obtenida con Kinect y demostró ser un método factible de aplicación en el campo de la rehabilitación.

Palabras clave: Biomecánica, Calibración funcional, Microsoft Kinect, Captura de movimiento.

\section{Abstract}

Biomechanical analysis is a tool to evaluate pathological movements and their rehabilitation. This paper examines the feasibility of improvements to the hip joint centre location in a Markerless Motion Capture System through the application of a functional calibration method. The movement of a young female with no physical impairments was analysed. Data taken from a gold standard system (Motion Capture System, MOCAP) and a low-cost alternative (Microsoft Kinect) were acquired simultaneously. Static and dynamic acquisitions were recorded. Five virtual markers for each leg were created from Kinect data and introduced into the optimal common shape technique and in the symmetrical centre of rotation estimation method to determine both hip joint centres. Results showed an improvement in joint centre location when compared to MOCAP information. In conclusion, this approach would improve the data obtained with Kinect and is shown to be a feasible method to apply in the rehabilitation field.

Keywords: Biomechanics, Functional calibration, Microsoft Kinect, Motion capture. 


\section{Introduction}

A powerful tool in the evaluation of movement dysfunction in orthopaedic and neurologic populations is the biomechanical analysis. The determination of joint kinematics during motion analysis frequently involves making certain assumptions regarding the point about which two segments move relative to one another. The determination of the centre of rotation (CoR) can often be difficult to measure in vivo, but knowledge of its exact location is important in a clinical gait analysis setting, where the calculation of hip joint moments may modify therapy (Ehrig et. al, 2011). Assessment of movement parameters, like gait, is commonly performed through high-end motion tracking systems or motion capture (MOCAP), which limits their measurement to sophisticated laboratory settings due to excessive costs.

Microsoft Kinect is a widely used alternative to marker-based motion capture systems due to its ease of use, portability, and low cost. Furthermore, Guzsvinecz et al. (2019), established that Kinect is the most accurate low-cost whole human body motion tracking sensor available in the market. This recent study defines accuracy as the degree of closeness of a measured quantity to its true value (Guzsvinecz et al, 2019).

Kinect has the potential to provide in-clinic assessments that support injury prevention, rehabilitation, and eldercare (Guess et al, 2017). Several investigations have validated the clinical utility of Kinect in the rehabilitation field, however, it has limitations and shows poor performance under certain circumstances (Guzsvinecz et al, 2019; Clark et al, 2019).

Some of the most important problems associated with depth sensors include occlusions or auto-occlusions in the image, unconventional postures, the use of wheelchairs or walkers, and noise within the "skeleton stream" (Mousavi Hondori and Khademi, 2014). The "skeleton stream" is a Microsoft Kinect SDK feature that determines the location and orientation of 25 joint centres in real-time. In order to improve the accuracy of this data, the use of Kalman filters, sensor fusion, and calibration have all been proposed (Mousavi Hondori and Khademi, 2014). Most of these techniques rely on software development, but researchers have noticed that both software and hardware are equally important factors in obtaining precise data (Guzsvinecz et al, 2019).

On the other hand, MOCAP systems have traditionally focused on the usage of skin markers. The reconstruction of skeletal kinematics, however, is limited by the relative motion of the skin markers over the underlying bones, an error referred to as soft tissue artefact. In this way, by generating a rigid marker configuration from the complete marker data of each segment, the optimal common shape technique (OCST) (Taylor et al, 2005) removes any motion of the markers relative to one another by minimising recorded artefacts. In combination with these techniques, functional approaches like the symmetrical centre of rotation estimation (SCoRE) (Ehrig et al, 2006), which identifies joint centres using the motion of one segment relative to another, have been proposed as key elements for increasing the accuracy in non-invasive determination of skeletal motion (Ehrig et al, 2011).

With these ideas in mind, the purpose of this study was to evaluate the feasibility of using skin markers techniques to compute joint centre locations, like OCST and SCoRE, in a markerless motion approach. A systematic and protocolised method was developed, and its results were compared with joint centre locations computed using a MOCAP system as gold standard.

In this pilot study, the hip joint centre location of one participant was analysed.

\section{Method}

\section{Participant and data collection}

Data from one healthy female subject without motor impairments or other disorders that could affect gait or motion (age $=34$ years, height $=1.66 \mathrm{~m}$, leg length $=0.87 \mathrm{~m}$ and weight $=66 \mathrm{~kg}$ ) was included. For this study, the coordinates of four pelvic and four thigh markers were defined. Each group of markers was arranged following the vertexes of a tetrahedron, conforming a marker cluster.

A hip motion of Star movement (Seven flexion-extension/abduction-adduction combined movements from the neutral position within the perimeter drawn in the Arc movement) followed by Arc movement (Flexion of $30^{\circ}$, half circumduction to an extension of $30^{\circ}$, neutral position) were performed according to (Camomilla et al, 2015). Six successful trials were recorded per subject so that within-subject ensemble averages could be calculated (Kadaba et al, 1989).

Motion data was collected using a 10-camera optical motion capture system running at $100 \mathrm{~Hz}$ (SMART-DX, BTS Bioengineering, Italy) to track reflective infrared markers attached to the test subject. Marker tracked data was low-pass filtered at a $6 \mathrm{~Hz}$ cutoff frequency using a dual-pass Butterworth digital filter.

Body motion was also simultaneously acquired with a single Kinect 2.0 (Kinect for XBOX ONE, Microsoft, Redmond, WA, USA). For its location, several considerations were taken based on the results of previous studies 
(Guzsvinecz et al, 2019; Chakraborty et al, 2020), as well as not to obstruct the field of view of the MOCAP's RGB cameras. The Kinect was turned on 20 min before the recording, and it was placed 2.5 meters in front of the subject to minimise the auto-occlusion. Figure 1 illustrates the set up.

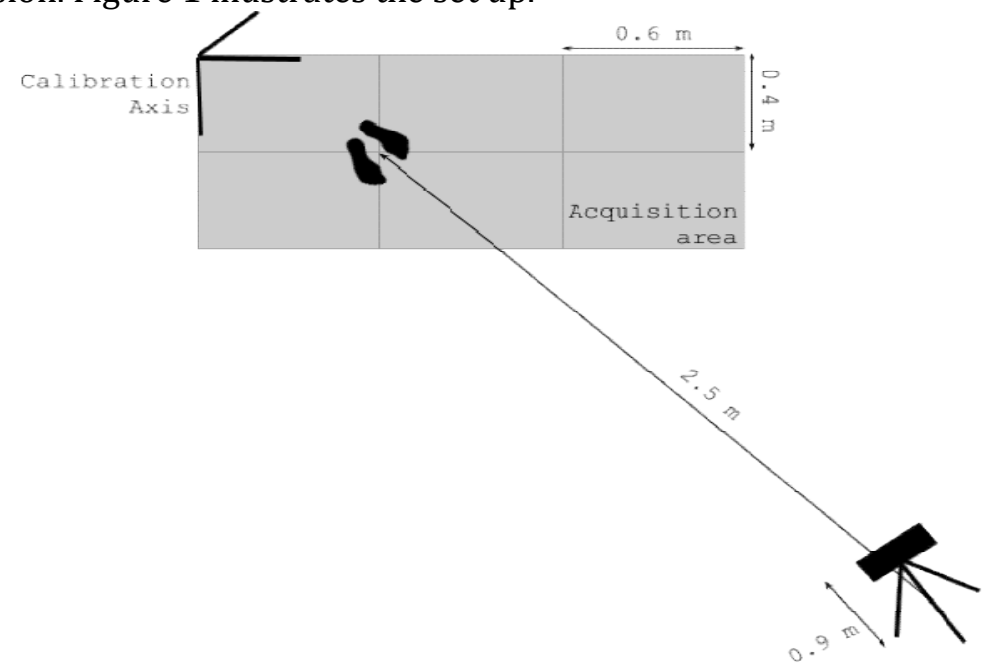

Fig. 1: Movement acquisition setup. A common acquisition area was delimited to determine the global coordinate system for both capture systems, i.e. MOCAP and Kinect

Kinect is a Markerless Motion Capture System (MMCS) with a depth sensor that acquires 3D information of the environment through a Time-of-Flight (ToF) method, which measures the speed of infrared light to calculate the distance. Kinect generates a different type of data for each arriving frame. The types of frames considered for this investigation were: depth frame (distance of each pixel to the camera), body index frame (pixel corresponding to a recognised body or not), and body frame (the so-called "skeleton stream" that represents the reconstructed joint centre location and orientation in 3D space).

\section{Review of the algorithms}

\section{Optimal common shape technique (OCST)}

The OSCT is an approach employed to reduce the effect of soft-tissue artefacts (Taylor et al, 2005) that uses the Procrustes analysis to determine the optimal common configuration of the markers, and to apply said configuration into each time frame. The ordinary Procrustes analysis fits one marker configuration $x_{i}$ of $i=1 \ldots n$ markers, into a second configuration $y_{i}$ by means of a rotation matrix $R$ and a translation vector $d$. This leads to the least-squares problem (Ec. 1)(Heller et al, 2011),

$$
\min _{R \in \Omega, d \in \mathbb{R}^{3}} \sum_{i=1}^{n}\left\|R x_{i}+d-y_{i}\right\|^{2} .
$$

that can be solved using singular value decomposition (Söderkvist and Wedin, 1993).

\section{Symmetrical centre of rotation estimation (SCoRE)}

SCoRE is an algorithm that determines the centre of rotation of spherical joints, based on the fact that a joint centre is stationary in each segment's local coordinate system (Ehrig et al, 2006). If the rotations $R_{i}$ and $S_{i}$, and translations $t_{i}$ and $d_{i}, i=1 \ldots n$, where $n$ is the total number of time frames, from the local segment coordinates to a global system for each time frame are known, the optimal $C o R$ can be obtained by solving the overdetermined linear least squares problem (Ec. 2),

$$
\min _{c_{1}, c_{2} \in \mathbb{R}^{3}} \sum_{i=1}^{n}\left\|R_{i} c_{1}+t_{i}-\left(S_{i} c_{2}+d_{i}\right)\right\|^{2},
$$

where $c_{1}$ and $c_{2}$ are the centres of rotation expressed in the local coordinate system of each segment linked at the joint centres. After a transformation into an appropriate global system, both centre representations $c_{1}=R_{i}$. $c_{1}+t_{i}$, $c_{2}=S_{i} \cdot c_{2}+d_{i}$ coincide for all time frames $i$, as long as the motion of the segments perfectly describes spherical circumvention around a common centre. 


\section{Functional calibration for Kinect}

The purpose of this study was to develop an acquisition protocol able to include OCST and SCoRE techniques in a markerless acquisition system. The protocol was designed around three components: the Microsoft Kinect sensor, a definition of virtual markers, and the OCST and SCoRE algorithms.

For the acquisition and processing of the information coming from the Kinect sensor, an ad-hoc software was developed in C\# in Visual Studio 2019 using Microsoft Kinect SDK V2.0. Kinect has a variable acquisition frequency based on events that can vary from 9 to 30 frames per second. For each frame recorded, an analysis of pixel data was performed.

For static acquisitions, one frame was analysed to generate virtual markers. In the computational modelling context used in gait analysis, a virtual marker is defined as a 3D point that aims to represent the position of a fictitious marker where there is none. To create said markers, the reconstructed thigh points were determined by analysing the information contained in the body index frame and the depth frame: the pixel should belong to a body (body index frame), and should be located in space ( $y$-coordinate) between the $y$-coordinate of the hip and the knee. From this set of points, five were selected for each leg. The position of five joints centres were obtained from the body frame ("skeleton stream"): spine base, right hip, left hip, right knee and left knee. The selected points were referenced to the corresponding knee joint centres to create virtual markers. The described process is detailed in Figure 2.

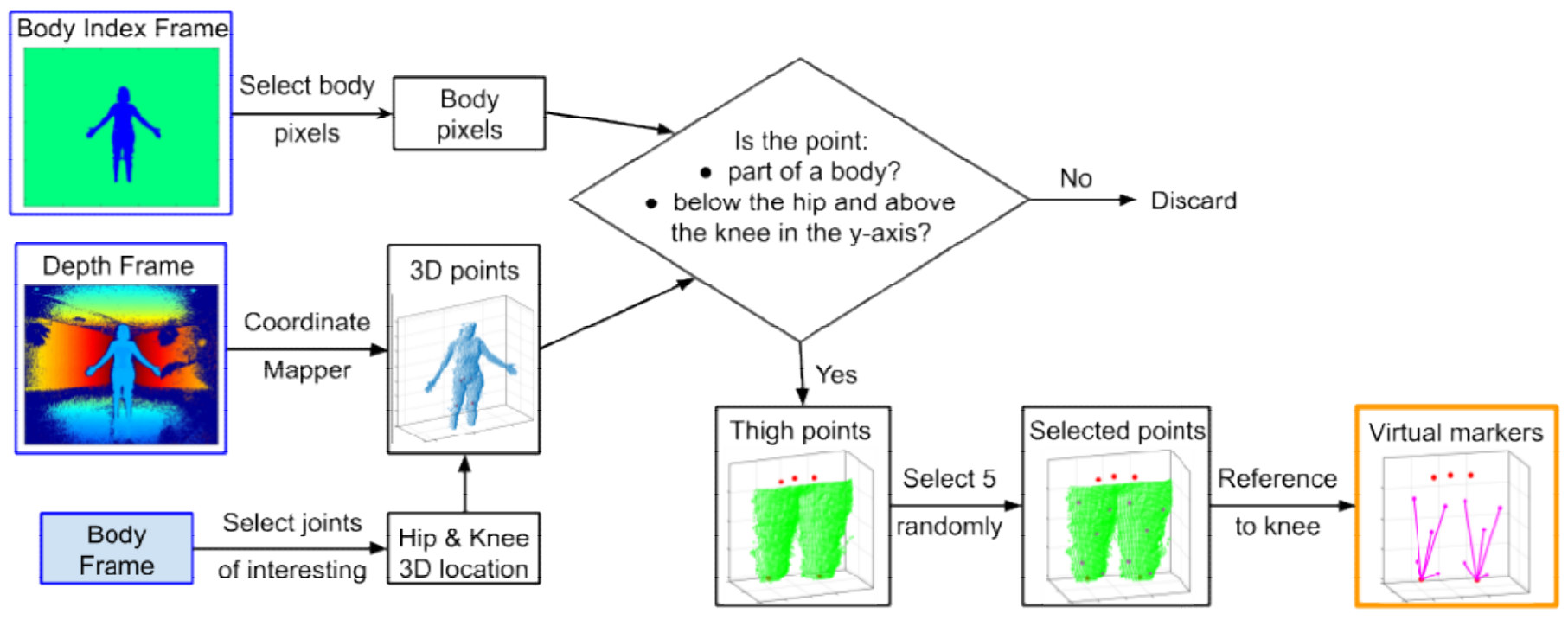

Fig. 2: Process for the creation of virtual markers. The Kinect data (input) is inside blue boxes. The result of the process is inside an orange box.

For dynamic acquisitions (functional calibration), the location and orientation of every joint centre was recorded for each frame. The virtual markers were created considering the points set from static acquisition by referencing them to the joint centre location.

Finally, in order to apply the functional calibration method to improve the hip joint centre location, the movement information of virtual markers created from Kinect data was piped into both OCST and SCoRE.

\section{Results}

Both depth and body index images obtained from Kinect are shown in Figure 3. The reconstructed 3D points are presented in Figure 4, where the points belonging to the femur segment are displayed in different colours. The red dots represent the location of the joint centres determined by Kinect. The virtual markers are painted in magenta, and referenced to the corresponding knee joint centre. For better representation, the joint centres and virtual markers have been enlarged. 

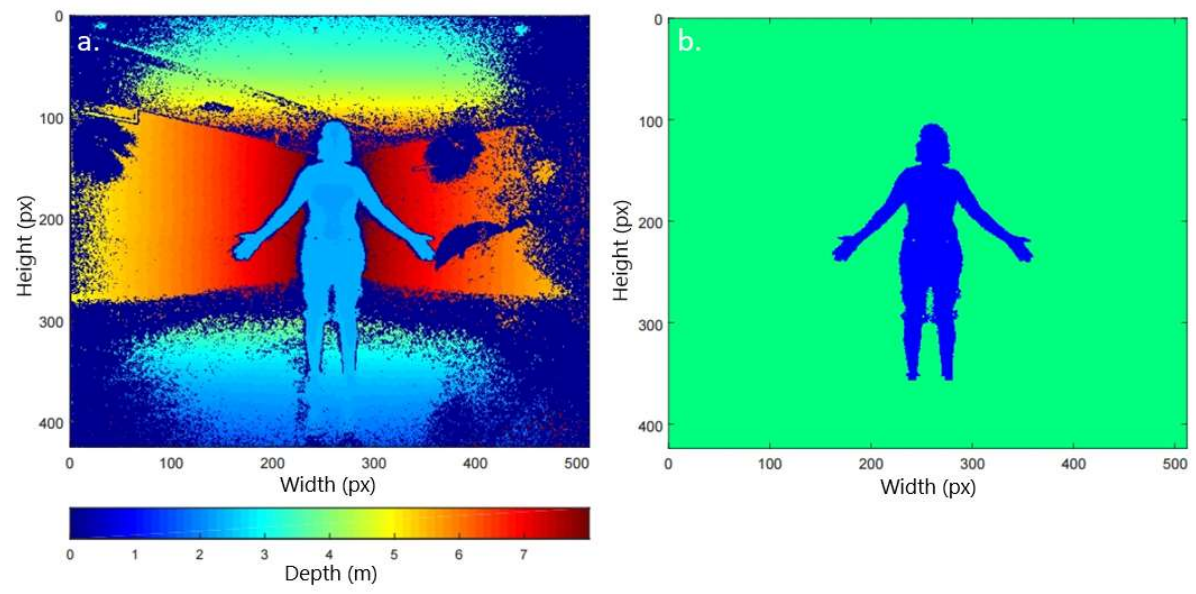

Fig. 3: Images obtained with Kinect. a.Depth Frame b.Body Index Frame.

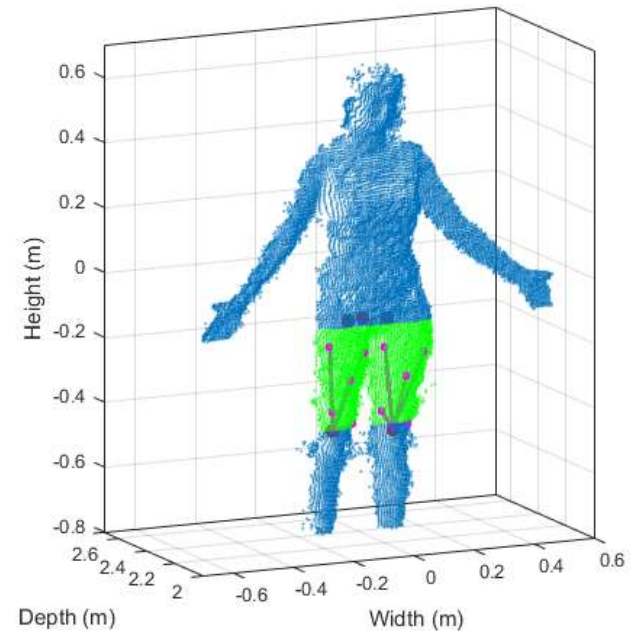

Fig. 4: 3D points reconstructed. The femur segment is coloured in green. In red dots, the joints location: spine base, left hip, right hip, left knee and right knee. In magenta, the virtual markers referenced to the corresponding knee.

Figure 5 shows the $x y z$-coordinate of the hip joint centre estimated by the Kinect sensor, the functional calibration for Kinect proposed in this study, and the gold standard (functional calibration with MOCAP data).

The functional calibration applied to Kinect data shows great improvements in the $x$-coordinate and $y$-coordinate of the joint centres, however, this approach did not provide a significant change in depth direction $z$-coordinate where the Kinect sensor has more limitations due to its ToF method.
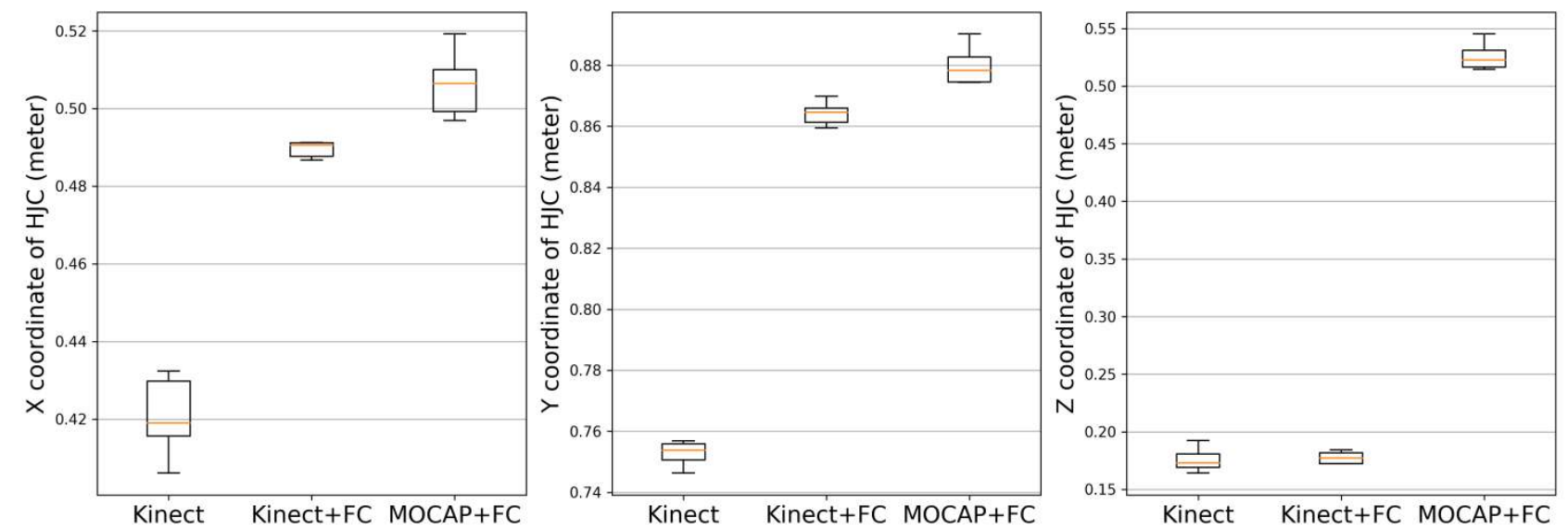

Fig. 5: Coordinates of HJC estimated by Kinect, the functional calibration for Kinect (Kinect+FC) and MOCAP (MOCAP+FC). Kinect sensor data was used as input of OCST and SCoRE algorithms.

\section{Conclusions}


A joint centre location technique of a markerless motion analysis system using a functional calibration method is presented in this paper. To carry out this technique and assess its feasibility, a pilot case of a young female with no physical impairments was analysed. Research applying functional calibration methods with Markerless Motion Capture System, Microsoft Kinect, has not been found in the literature. In contrast, the functional calibration is a widely used method to determine the joint centre location with MOCAP systems (Camomilla et al, 2015). The novelty of this study resides in the incorporation of a calibration method seeking to increase the accuracy of the location of the tracked joints by Kinect in global coordinates. The protocol used to capture the data was based on different publications (Guzsvinecz et al, 2019; Chakraborty et al, 2020), and it turned out to be feasible in the rehabilitation field. All the data needed for a functional calibration can be obtained in few, short acquisitions, and any subsequent data processing can be carried out using most readily available computers or laptops, which makes it suitable for clinical applications.

The Kinect results were compared with the MOCAP results, showing an improvement in the measurement of hip centre locations. Nevertheless, a more detailed study of the effect of Kinect sensor position on the estimation of depth coordinate is required. This early advance encourages further investigation of this method, possibly by increasing the number of studied subjects and evaluated joints.

\section{References}

Camomilla, V., Bonci, T., Dumas, R., Chèze, L., and Cappozzo, A. (2015). A model of the soft tissue artefact rigid component. Journal of Biomechanics, 48(10):1752-1759.

Chakraborty, S., Nandy, A., Yamaguchi, T., Bonnet, V., and Venture, G.(2020). Accuracy of image data stream of a markerless motion capture system in determining the local dynamic stability and joint kinematics of human gait. Journal of Biomechanics, page109718.

Clark, R. A., Mentiplay, B. F., Hough, E., and Pua, Y. H. (2019). Three-dimensional cameras and skeleton pose tracking for physical function assessment: A review of uses, validity, current developments and kinect alternatives. Gait and Posture, 68:193-200.

Ehrig, R. M., Heller, M. O., Kratzenstein, S., Duda, G. N., Trepczynski, A., and Taylor, W. R. (2011). The SCoRE residual: A quality index to assess the accuracy of joint estimations. Journal of Biomechanics, 44(7):1400-1404.

Ehrig, R. M., Taylor, W. R., Duda, G. N., and Heller, M. O. (2006). A survey of formal methods for determining the centre of rotation of ball joints.Journal of Biomechanics,39(15):2798-2809.

Guess, T. M., Razu, S., Jahandar, A., Skubic, M., and Huo, Z. (2017). Comparison of 3D Joint Angles Measured With the Kinect 2.0 Skeletal Tracker Versus a Marker Based Motion Capture System. Journal of Applied Biomechanics, 33(2):176-181.

Guzsvinecz, T., Szucs, V., and Sik-Lanyi, C. (2019). Suitability of the kinect sensor and leap motion controller - a literature review. Sensors, 19(5):1072.

Heller, M. O., Kratzenstein, S., Ehrig, R. M., Wassilew, G., Duda, G. N., andTaylor, W. R. (2011). The weighted optimal common shape technique improves identification of the hip joint center of rotation in vivo. Journal of Orthopaedic Research, 29(10):1470-1475.

Kadaba, M., Ramakrishnan, H., Wootten, M., Gainey, J., Gordon, G., and Cochran, G. (1989). Repeatability of kinematic, kinetic, and electromyographic data in normal adult gait. Journal of Orthopaedic Research, 7:849-860.

Mousavi Hondori, H. and Khademi, M. (2014). A review on technical and clinical impact of microsoft kinect on physical therapy and rehabilitation. Journalof Medical Engineering, 2014.

Söderkvist, I. and Wedin, P. Å. (1993). Determining the movements of the skeleton using well-configured markers. Journal of Biomechanics, 26(12):1473-1477.Functional calibration for Kinect9

Taylor, W., Ehrig, R. M., Duda, G. N., Schell, H., Seebeck, P., and Heller, M. O.(2005). On the influence of soft tissue coverage in the determination of bone kinematics using skin markers. Journal of Orthopaedic Research, $23: 726-734$ 